\title{
Zone plate tilt study in transmission X-ray microscope system at 8-11
}

\author{
$\mathrm{keV}$ \\ Fu-Han Chao ${ }^{1,2}$, Gung-Chian Yin ${ }^{2}$, Keng. S Liang ${ }^{2}$, and Yin-Chieh Lai ${ }^{1}$ \\ ${ }^{1}$ Department of Photonics, National Chiao Tung University, Hsinchu 30076, Taiwan \\ ${ }^{2}$ National Synchrotron Radiation Research Center, Hsinchu 30076, Taiwan
}

\begin{abstract}
Zone plate [1] has been used as a focal lens in transmission X-ray microscope (TXM) optical system in recent decades $[2,3]$. In TXM of NSRRC[4,5], the thickness of zone plate is about $900 \mathrm{~nm}$ and the width of its out most zones is $50 \mathrm{~nm}$, which has a high aspect ratio 18 . When zone plate is tilted, the image quality will be affected by aberration. Since the aspect ratio of zone plate is large, for incident beam, the shape of zone plate's transmission function will look different when zone plate is tilted.
\end{abstract}

The both experimental and simulation result will be shown in this present. A five axes stage is designed and manufactured for the zone plate holder for three dimensional movement, tip and tilt. According to Fourier theory, we can calculate the wave distribution on image plane, if we know the original wave function, the distances between each element, and the transparencies of the sample and zone plate. A parallel simulation process code in MATLAB is developed in workstation cluster with up to 128 Gbytes memory. The effects of aberration generated by tilt effect are compared from the experimental data and simulation result. A maximum tilt angle within the acceptable image quality is calculated by simulation and will be verified by experiment.

\section{Keyword list}

Zone plate, transmission X-ray microscope (TXM), aberration

\section{INTRODUCTION}

Transmission X-ray microscope (TXM) is one of the X-ray microscopes. NSRRC's TXM was designed and installed in September 2004 by NSRRC and Xradia, it was the first zone plate based hard X-ray microscope operated at 8-11 keV. The resolution of this TXM is about $60 \mathrm{~nm}$ in hard X-ray region. Since the experiment and simulation of zone plate tilt was performed in this end station, the structure of the TXM system and the zone plate in TXM system will be introduced in this chapter.

Advances in X-Ray/EUV Optics and Components IV, edited by Ali M. Khounsary, Christian Morawe, Shunji Goto, Proc. of SPIE Vol. 7448, 74480X · C 2009 SPIE · CCC code: 0277-786X/09/\$18 · doi: 10.1117/12.826723 


\section{Sec. 1.1 The structure of TXM system}

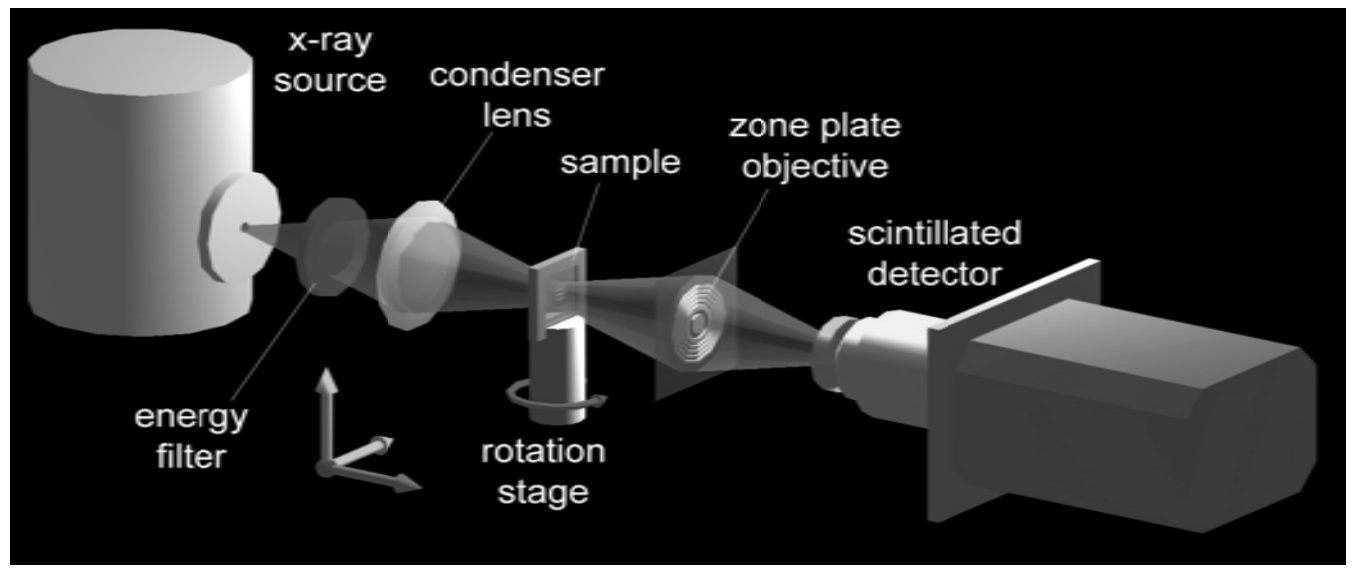

Fig. 1-1 The structure of TXM system. The zone plate can be regarded as a X-ray lens.

The structure of TXM system is shown in Fig. 1-1. X-ray come out from the synchrotron, pass through the energy filter and the condenser. Photons with energy from 8 to $11 \mathrm{keV}$ are filtered through the energy filter. After passing through the condenser, X-rays become convergent, hollow cone, and focused at the sample. The zone plate which is put behind the sample acts as an objective lens in microscope. In the scintillated system, the scintillator converts X-ray to visible light, and the CCD gets the image.

\section{Sec. 1.2 Zone plate in TXM system}

In TXM system, the zone plate is made of gold on the silicon nitride membrane by electron beam lithography, it acts as a focal lens. The distance between the zone plate and the sample is equal to the focal length of zone plate, $27 \mathrm{~mm}$. And the zone plate of 75 to $85 \mu m$ diameter is well illuminated by the hollow cone beam from the condenser. A zone plate consists of many concentric rings. Light passing through the zone plate will diffract around the rings. And the zone plate uses diffraction to focus light. The zone plate has a diffraction-limited resolution $\delta_{\mathrm{m}}=\mathrm{K}_{1} \lambda / \mathrm{NA}=2 \mathrm{~K}{ }_{1} \mathrm{dr} / \mathrm{m}$, where $\mathrm{m}$ is the diffraction order, and $\mathrm{dr}$ is the width of the outmost ring, $0.3 \leq K_{1} \leq 0.61$, depending on the illumination condition. In TXM system, $K_{1}=0.61$, and the outmost ring width dr of the zone plate is $50 \mathrm{mn}$, so the spatial resolution of first order is about $60 \mathrm{~nm}$. By the way, the aspect ratio of zone plate is defined as:

$$
\text { Aspect ratio } \equiv \frac{h}{d r}
$$

, where $\mathrm{h}$ is the thickness of zone plate. In order to achieve phase shift of $\pi / 2$ at $8 \mathrm{keV}$, the thickness of gold has to be about $890 \mathrm{~nm}$. The aspect ratio is about 18 . However, the optimal thickness of zone plate is about $1.8 \mu \mathrm{m}$ to achieve a phase shift of $\pi$ at $8 \mathrm{keV}$ (the aspect ratio is about 36). Since the achievable aspect ratio for gold zone plate was about 18 when TXM is installed, the fabrication of an ideal zone plate which can achieve a phase shift of $\pi$ is still a big challenge.

The zone plate in TXM system is a phase zone plate, and the transmission function of a phase zone plate can be expressed as [1]: 


$$
T(x, y)=\exp \left\{-i \pi\left[\frac{1}{2}+\frac{2}{\pi} \sum_{m=1,3,5 \ldots}^{\infty} C_{m} \sin \left(m \pi \frac{x^{2}+y^{2}}{\lambda f}\right)\right]\right\}
$$

, where $\mathrm{m}$ is the diffraction order, $\mathrm{f}$ is the focal length of zone plate, $\lambda$ is the wavelength, and $\mathrm{C}_{\mathrm{m}}=1 / \mathrm{m}$ is the weight of each diffraction number. The term $1 / 2$ represents the non-diffraction zero order background, and $m=1,3,5, \ldots$ represent for the first, the third, the fifth order, etc.

\section{THEORY}

In this chapter, the calculation of X-ray optical fields in TXM system is based on the paraxial approximation in scalar wave field theory [6]. The approximation requires that the propagation distances are much larger than the wavelength and that the sample and zone plate can be described by thin element transmission functions. Besides, quasi-monochromatic object illumination conditions are assumed. We set the wave length of X-ray to be 1.5 angstrom (the photon energy is about $8.27 \mathrm{keV}$ ) in our calculation and simulation.

\section{Sec. 2.1 Zone plate tilt}

The shape of zone plate's transmission function will look different for incident beam when zone plate is tilted. The relationship between the tilt angle and the effective transmission function of the zone plate will be discussed. In this section, $\mathrm{T}$ represents the original transmission function of a zone plate, $T^{\prime}$ represents the effective transmission function of the tilted zone plate, and $\theta$ is the tilt angle. For phase zone plate, both the absorption and the phase shit are related to the optical path length in the zone plate. As the optical path length increases, the absorption and the phase retardation due to zone plate increase.

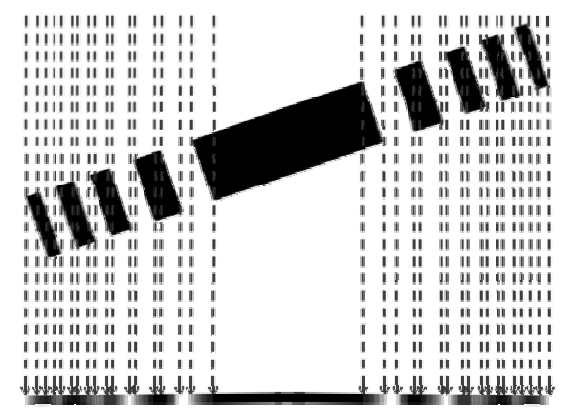

Fig. 2-1 The schematic of projection of a tilted opaque zone plate. The optical path length in zone plate has to be considered.

The optical path length in zone plate decreases gradually on the edge of rings, as shown in Fig. 2-1, so the projection is blurred on the edge. And when tilt angle $\theta \geq \tan ^{-1}\left(\frac{1}{\text { aspect ratio }}\right) \cong \frac{1}{\text { aspect ratio }}$, the projections of outmost rings will overlap.

\section{Sec. 2.1.1 Calculation of the effective transmission function}


Taking the relationship between the transmission and the optical path length into account will make the calculation of the effective transmission function to be more complicated. In order to solve this problem, we consider the zone plate as a set of thin zone plates with transmission functions $T_{1}, T_{2}, \ldots, T_{n}$, as shown in Fig. 2-2.

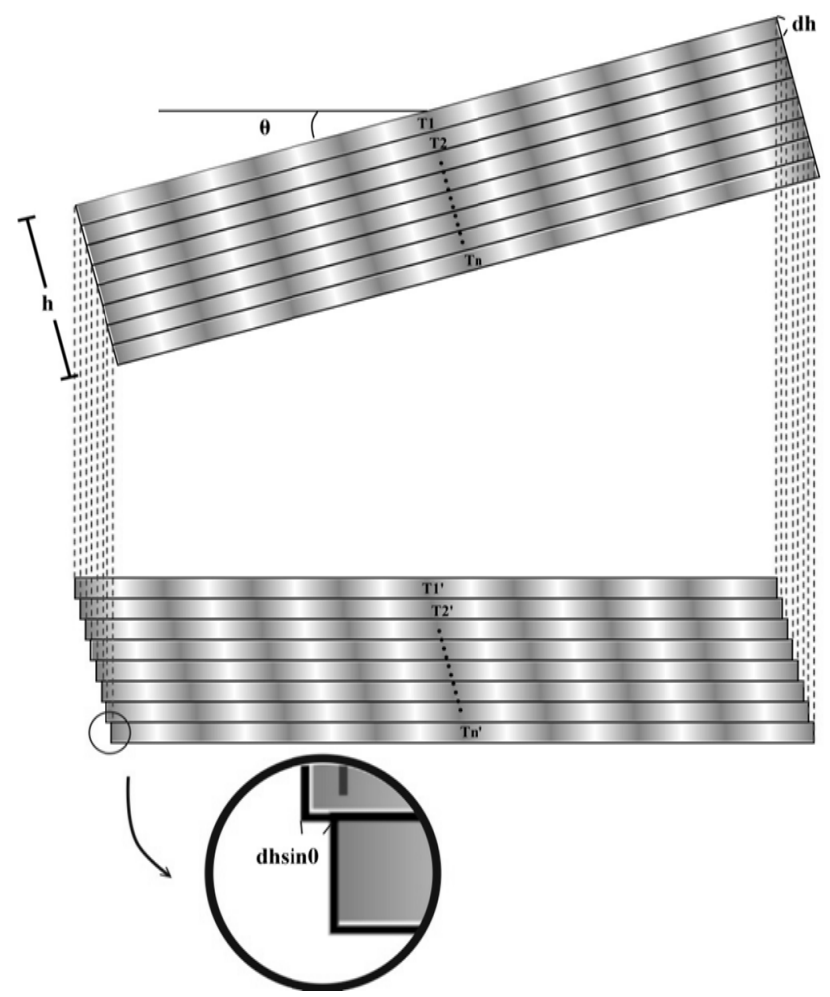

Fig. 2-2 Regard the tilted zone plate as many thin zone plates, where $T_{1}, T_{2}, \ldots T_{n}$ are the transmission functions of the thin zone plates. $\mathrm{h}$ is the thickness of the zone plate, and $\mathrm{dh}$ is the thickness of the thin zone plates. The effective transmission functions $T_{1}{ }^{\prime}, T_{2}{ }^{\prime}, \ldots . ., T_{n}{ }^{\prime}$ are the projections of $\mathrm{T}_{1}, \mathrm{~T}_{2}, \ldots \mathrm{T}_{\mathrm{n}}$, respectively. And the projections are shifted by $d h \sin \theta_{\text {from each other. }}$

The transmission functions of zone plate $\mathrm{T}$ can be expressed as:

$$
T=T_{1} \cdot T_{2} \cdot \ldots . . \cdot T_{n}=\prod_{m=1}^{n} T_{m}
$$

We can get the projections of those thin zone plate separately, and the effective transmission functions of the thin zone plates are expressed as $T_{1}{ }^{\prime}, T_{2}{ }^{\prime}, \ldots \ldots, T_{n}{ }^{\prime}$. The relationship between $\mathrm{T}_{\mathrm{m}}$ and $T^{\prime}{ }_{\mathrm{m}}$ is:

$$
T_{m}{ }^{\prime}(x, y)=T_{m}\left(x^{\prime}, y^{\prime}\right)
$$

, where $\mathrm{m}=1,2, \ldots, \mathrm{n} . \quad x^{\prime}=\left[x+\left(m-\frac{n}{2}\right) \cdot d h \sin \theta\right] \cdot \sec \theta$, and $y^{\prime}=y$. Multiple the effective transmission functions, and then we can get the effective transmission function T', i.e. 


$$
T^{\prime}=T_{1}{ }^{\prime} \cdot T_{2}{ }^{\prime} \cdot \ldots . . \cdot T_{n}{ }^{\prime}=\prod_{m=1}^{n} T_{m}{ }^{\prime}
$$

By eq. (2.2), we can expect that, the focal lengths $f_{x}{ }^{\prime}$ and $f_{y}{ }^{\prime}$ of the effective transmission function in horizontal and vertical directions will be

$$
f_{x}^{\prime}=\frac{f}{\sec ^{2} \theta}=f \cos ^{2} \theta, f_{y}^{\prime}=f
$$

Since $\cos \theta \leq 1, f_{x}^{\prime}$ is shorter than $f$. So we can expect that the TXM image will be affected by astigmatism when the zone plate is tilted.

\section{Sec. 2.1.2 The cross sections of tilted zone plate}

The effective transmission function of tilted zone plate can be solved by eq. $(2,1 \sim 3)$, and the cross sections (the optical path length in zone plate v.s the radius) of the effective transmission functions at different tilt angles are shown in Fig. 2-3.
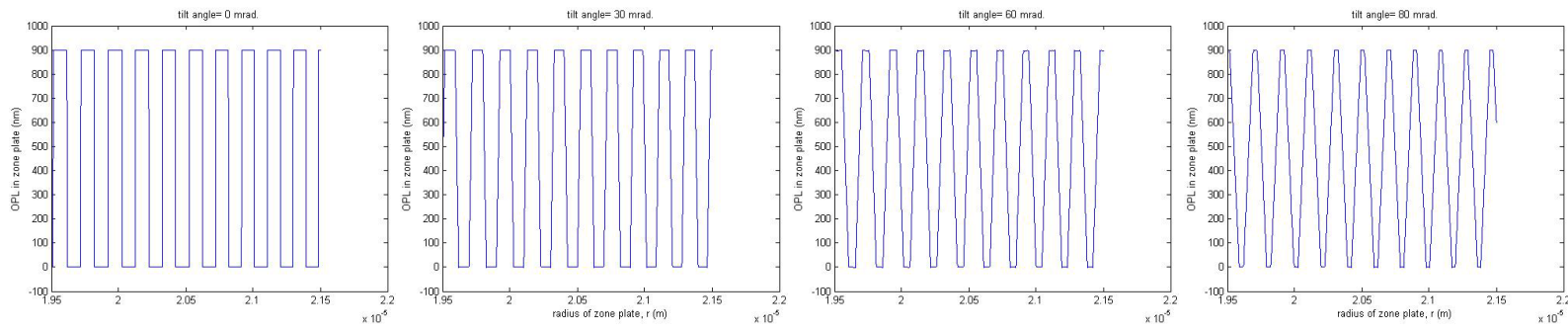

(a)
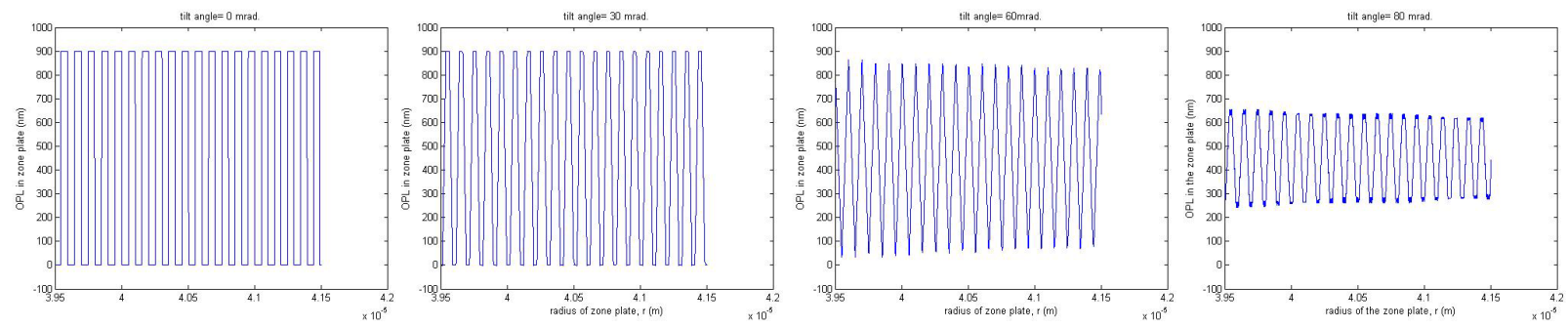

(b)

Fig. 2-3 The optical path length in zone plate v.s the radius of zone plate at tilt angle $\theta=0,30,60,80 \mathrm{mrad}$. (a) around $\mathrm{r}=20 \mu \mathrm{m}$ (dr is about $100 \mathrm{~nm}$, the respect ratio is about 9). (b) around $\mathrm{r}=40 \mu \mathrm{m}(\mathrm{dr}$ is about $50 \mathrm{~nm}$, the respect ratio is about 18$)$.

In Fig. 2-3, at tilt angle $\theta=0 \mathrm{mrad}$, the cross sections are composed of many rectangular functions. The edges of the

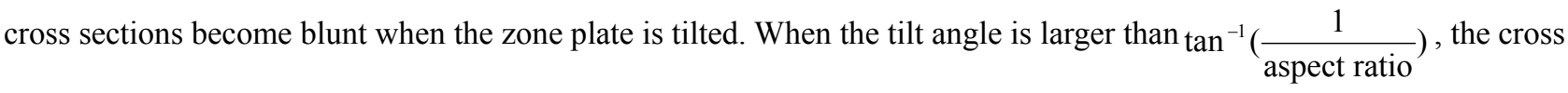
section is approximately triangular in shape. As a result, we can expect that, the ability of zone plate to focus light in horizontal direction becomes worse when the tilt angle is too large. 


\section{Sec. 2.2 Wave propagation}

The optical fields in TXM system can be calculated by Fourier optics theory [4].

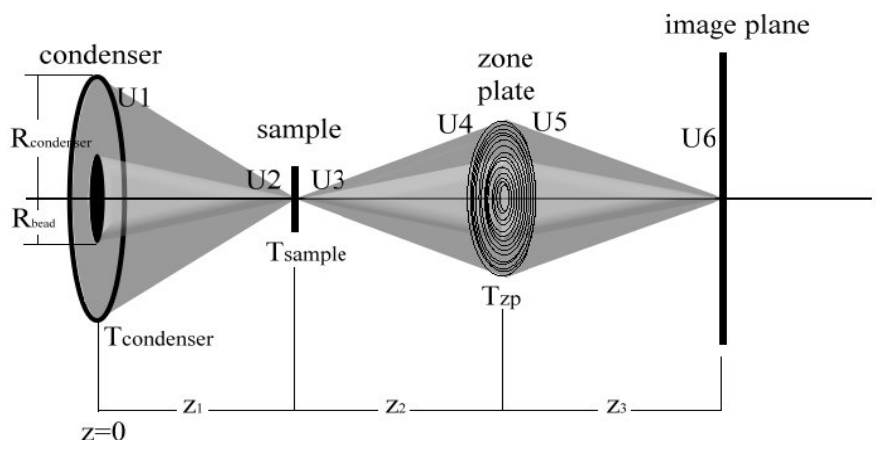

(a)

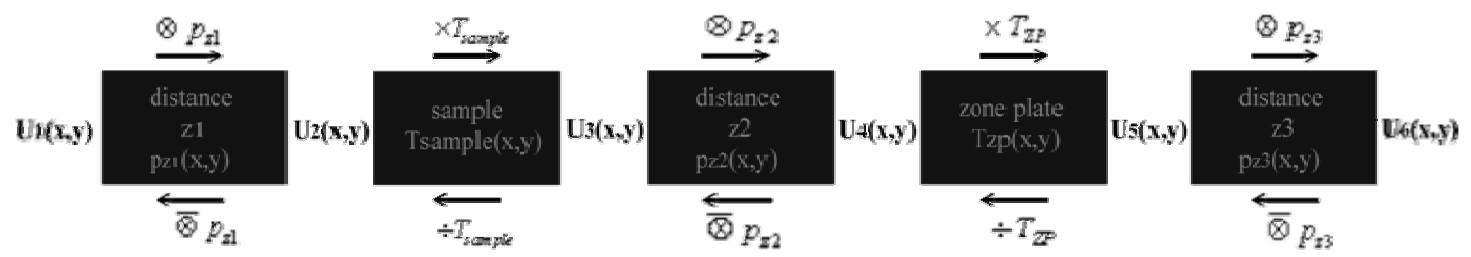

(b)

Fig. 2-4 (a) Optical wave propagates from the condenser to the image plane. The distances between each element are $z_{1}, z_{2}, z_{3}$, and $\frac{1}{z_{2}}+\frac{1}{z_{3}}=\frac{1}{f}, \mathrm{f}$ is the focal length of the zone plate. $\mathrm{U}_{1}, \mathrm{U}_{2}, \mathrm{U}_{3}, \mathrm{U}_{4}, \mathrm{U}_{5}, \mathrm{U}_{6}$ are the optical fields at $\mathrm{z}=0, \mathrm{z}=\mathrm{Z}_{1}^{-}, \mathrm{z}=\mathrm{z}_{1}^{+}, \mathrm{z}=\left(\mathrm{z}_{1}+\mathrm{z}_{2}\right)^{-}$, $\mathrm{z}=\left(\mathrm{z}_{1}+\mathrm{z}_{2}\right)^{+}, \mathrm{z}=\mathrm{Z}_{1}+\mathrm{z}_{2}+\mathrm{z}_{3}$, respectively. $\mathrm{T}_{\text {condenser }}, \mathrm{T}_{\text {sample }}, \mathrm{T}_{\mathrm{ZP}}$ are the effective transmission functions of the condenser, the sample and the zone plate. (b) Illustration of optical fields calculation, where “ $\bigotimes$ ” and " $₫$ ” denote convolution and de-convolution.

A part of the TXM system (from the condenser to the image plane of the zone plate), and the optical fields at many positions are shown in Fig. 2-4. $U_{1}$ is the optical field at the output of the condenser, it's convergent, hollow cone beam. The wave function $\mathrm{U}_{1}$ can be calculated by multiplying a radial symmetric, oblique light wave $\mathrm{U}_{0}$ by the transparency $\mathrm{T}$ condenser. That is,

$$
\begin{gathered}
U_{1}(x, y)=U_{0}(x, y) \cdot T_{\text {condenser }}(x, y) \\
U_{0}(x, y)=A \cdot \exp \left(j k_{r} \sqrt{x^{2}+y^{2}}\right)=U_{0}(r)=A \cdot \exp \left(j k_{r} r\right)
\end{gathered}
$$

, where $\mathrm{k}_{\mathrm{r}}=\mathrm{k} \cos (0.87 \mathrm{mrad})$ and

$$
T_{\text {condenser }}(x, y)=\left\{\begin{array}{l}
1, R_{\text {bead }}<r=\sqrt{x^{2}+y^{2}}<R_{\text {condenser }} \\
0, \text { otherwise }
\end{array}\right.
$$

According to the Fresnel diffraction integral and the convolution theorem [4], the optical field $U_{2}$ is equal to the convolution of $\mathrm{U}_{1}$ and Fourier propagator $\mathrm{p}_{\mathrm{z} 1}$ :

$$
U_{2}(x, y)=U_{1}(x, y) \otimes p_{z 1}(x, y)=F^{-1}\left\{U_{1}\left(f_{x}, f_{y}\right) \cdot P_{z 1}\left(f_{x}, f_{y}\right)\right\}
$$


, where

$$
p(x, y, z)=\frac{\exp (j k z)}{j \lambda z} \cdot \exp \left[k \cdot\left(x^{2}+y^{2}\right) / 2 z\right]
$$

, and $p_{z 1}(x, y)=p\left(x, y, z_{1}\right)$.

We can calculate the optical field $\mathrm{U}_{3}$ by multiple $\mathrm{U}_{2}$ and the transparency of the sample $\mathrm{T}_{\text {sample }}$.

$$
U_{3}(x, y)=U_{2}(x, y) \cdot T_{\text {sample }}(x, y)
$$

Similarly, the optical fields $U_{4}, U_{5}$ and $U_{6}$ can be calculated by Fourier optics theory, the illustration of the calculation is shown in Fig. 2-4 (b), where $\mathrm{p}_{\mathrm{z} 2}(\mathrm{x}, \mathrm{y})=\mathrm{p}\left(\mathrm{x}, \mathrm{y}, \mathrm{z}_{2}\right)$ and $\mathrm{p}_{\mathrm{z} 3}(\mathrm{x}, \mathrm{y})=\mathrm{p}\left(\mathrm{x}, \mathrm{y}, \mathrm{z}_{3}\right)$.

\section{SIMULATION}

The theories of wave propagation and zone plate tilt were introduced in chapter 2, and there are some simulation results in this chapter. A Matlab program is designed to calculate the optical fields in TXM system, and it also can calculate the effective transmission function of a tipped / tilted zone plate. There are some simulated images acquired at different tip / tilt angles, the images will be compared and the relationship between the image quality and the tip / tilt angle will be discussed.

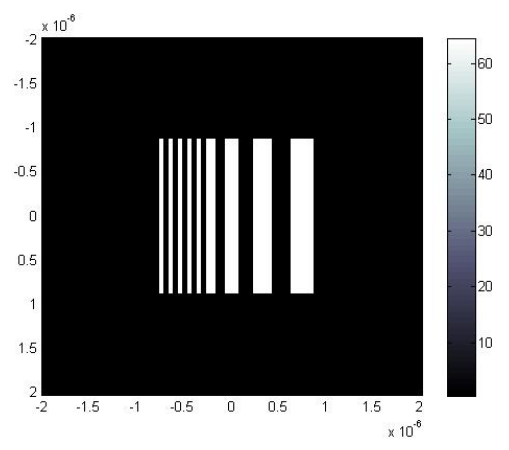

Fig. 3-1 The transparency of the test sample. There are 9 vertical lines, the width of the thinnest line is $50 \mathrm{~nm}$.

The simulated images are shown in Table 3-1:

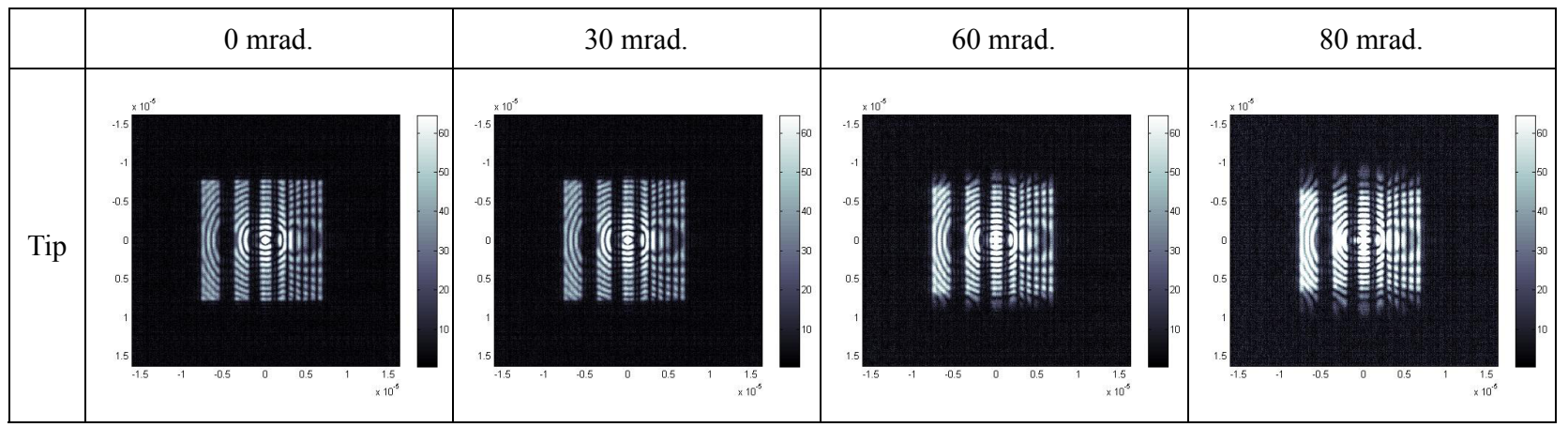




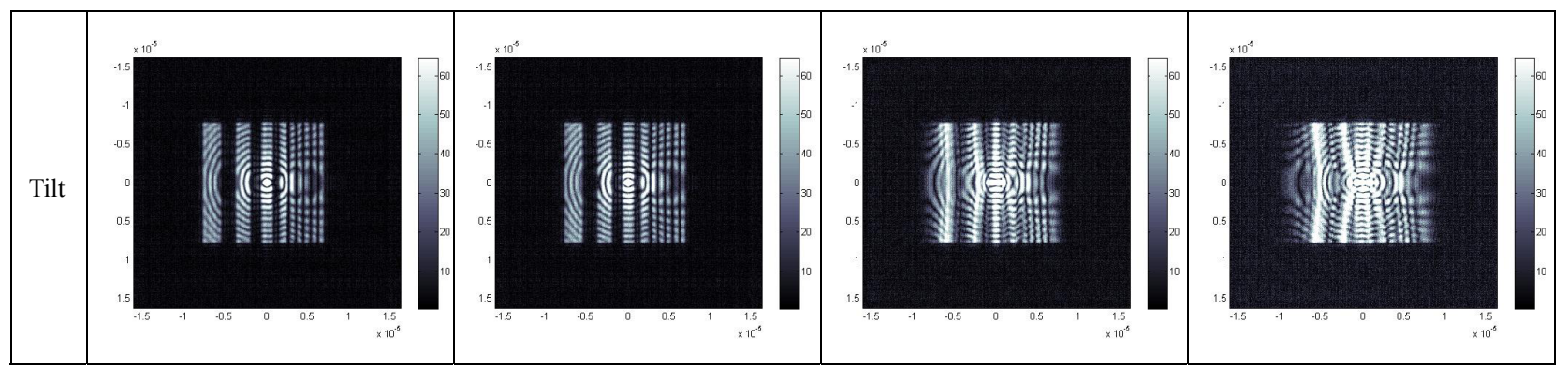

Table 3-1 The simulated images are gotten when the zone plate is tipped /tilted by 0, 30, 60, 80 mrad.

Compare the images in Table 3-1, we can see that, the images will be compressed in vertical direction when the zone plate is tipped, and they will be compressed in horizontal direction when the zone plate is tilted. Besides, the last four simulated images in Table 3-1 are evidently distorted. The normalized amplitude line plots of these simulated images are shown in Fig. 3-2. In Fig. 3-2, Cx represents the normalized amplitude line plot in x direction, and Cy represents the normalized amplitude line plot in y direction.

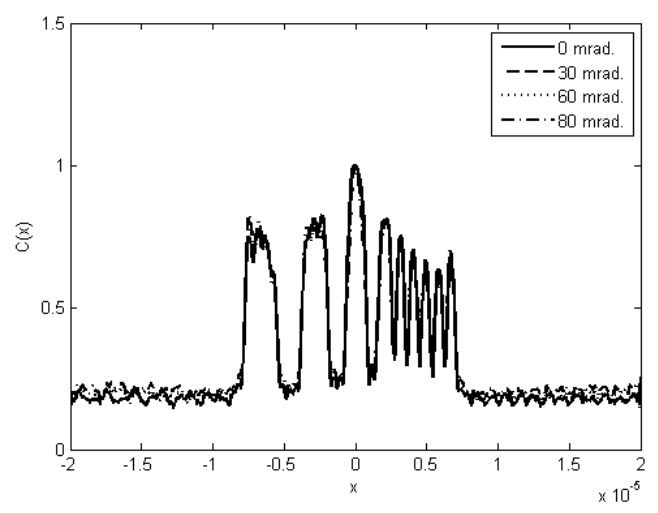

(a)

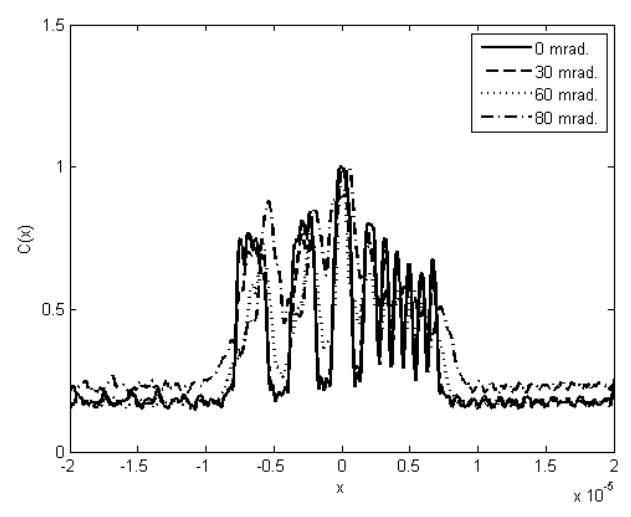

(b)

Fig. 3-2 (a) The line plots Cx when the zone plate is tipped by 0, 30, 60, and 80 mrad. (b) The line plots Cx when the zone plate is tilted by $0,30,60$, and $80 \mathrm{mrad}$.

The line plots of different tip angle are almost the same in Fig. 3-2 (a). That is, the amplitude distributions of $\mathrm{U}_{6}$ in $\mathrm{x}$ direction won't change evidently when the zone plate is tipped. However, the line plots o in Fig. 3-2 (b) are quite different. In Fig. 3-2 (b), the edges of the pattern get blunter, and the contrasts become worse as the tilt angle increases. Compare Fig. 3-2 (a) and (b), and calculate the contrast modulations. The relationships between the contrast modulations and the tip / tilt angles are shown in Fig. 3-3. By Fig. 3-3, we can see that, the contrast modulation of TXM image doesn't change obviously as the tip angle increases. Nevertheless, the contrast modulation decreases as the tilt angle increases. 


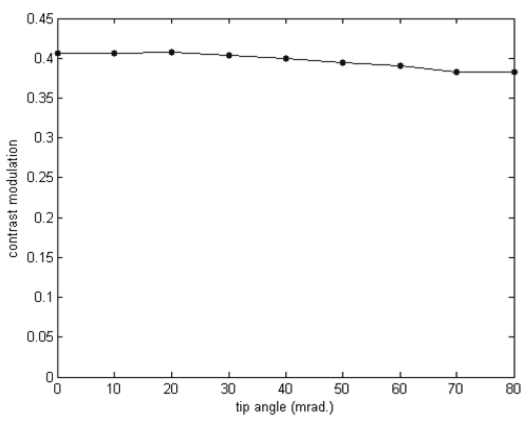

(a)

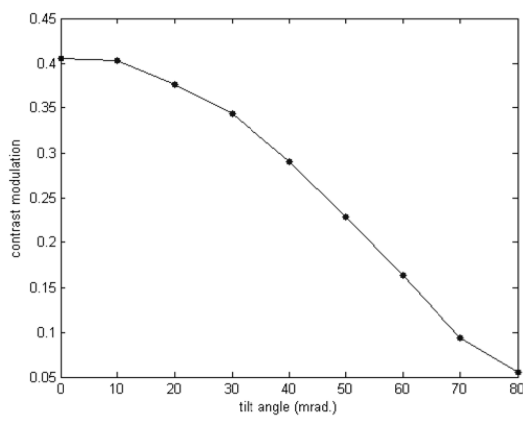

(b)

Fig. 3-3 (a) Contrast modulation v.s tip angle, and (b) contrast modulation v.s tip angle at spatial frequency $v=\frac{1}{50 \mathrm{~nm}}=20 \mathrm{MHz}$.

\section{EXPERIMENTAL RESULTS}

In chapter 3, the TXM images with different tip / tilt angles were obtained by simulation. The experimental results of zone plate tilt in TXM system will be shown in this chapter.

\section{Sec. 4.1 The Experiment of Zone Plate Tilt}

\section{Sec. 4.1.1 Five Axes Stage in TXM System}

In TXM system, the zone plate holder is put on a five axes stage. The stage was designed and manufactured by Newport company, called AG-M050, as an optical mount, as shown below in Fig. 4-1. It can move zone plate in $\mathrm{x}, \mathrm{y}$, and $\mathrm{z}$ directions, tip and tilt zone plate (The angular range of AG-M050 is about $\pm 2 \mathrm{deg} . \cong \pm 34.9 \mathrm{mrad}$., the maximum tip / tilt angle of our five axes stage is about $40 \mathrm{mrad}$.). There is a piezoelectric crystal in the stage to adjust the tip / tilt angle. Experiments of zone plate tilt can be done with this stage.

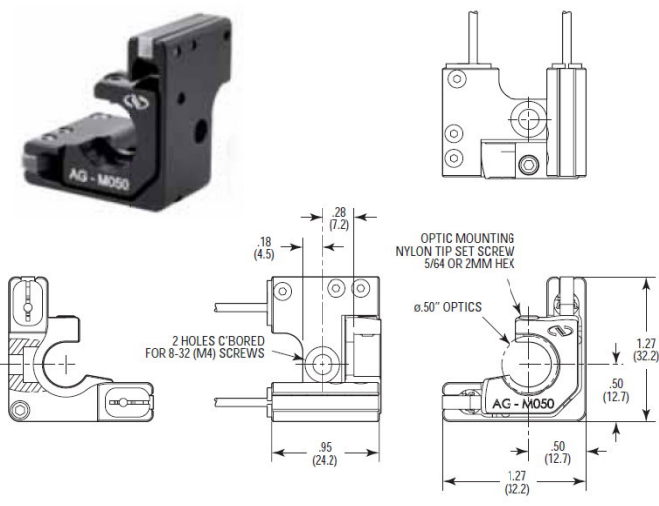

(a)

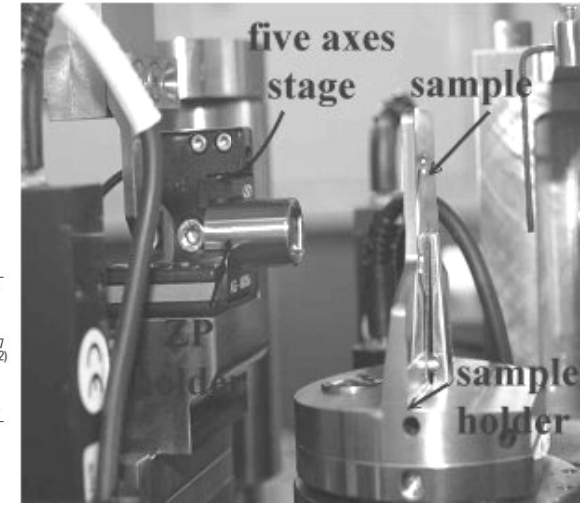

(b)

Fig. 4-1 (a) Model AG-M050. (b) Five axes stage of the zone plate holder in TXM system. 


\section{Sec. 4.1.2 The Siemens star}

The Siemens star is also called the spoke pattern and it is a device to test the resolution of optical instruments. This pattern is made by Yu-Tong Chen [10].The line width of the spoke pattern is narrower in the inner part than in the outer part. A spoke pattern was used to be the test sample in the zone plate tip / tilt experiment. For the Siemens star which was used in our experiment, the width of the pattern in each zone was between $40 \mathrm{~nm}$ to $50 \mathrm{~nm}, 50 \mathrm{~nm}$ to $60 \mathrm{~nm}, 60 \mathrm{~nm}$ to 70 $\mathrm{nm}, 70 \mathrm{~nm}$ to $80 \mathrm{~nm}, 80 \mathrm{~nm}$ to $90 \mathrm{~nm}, 90 \mathrm{~nm}$ to $100 \mathrm{~nm}$ (from the inner most zone to the out most zone). Fig. 4-2 shows the TXM image of the Siemens star which was obtained without tilting the zone plate:

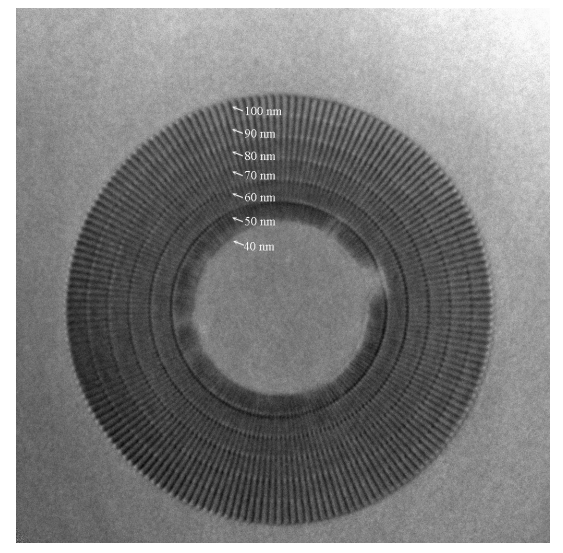

Fig. 4-2 The TXM image of the Siemens star.

\section{Sec. 4.2 Experimental Data and Line Plots}

The experimental results are shown in Table 4-1 (a) (e):

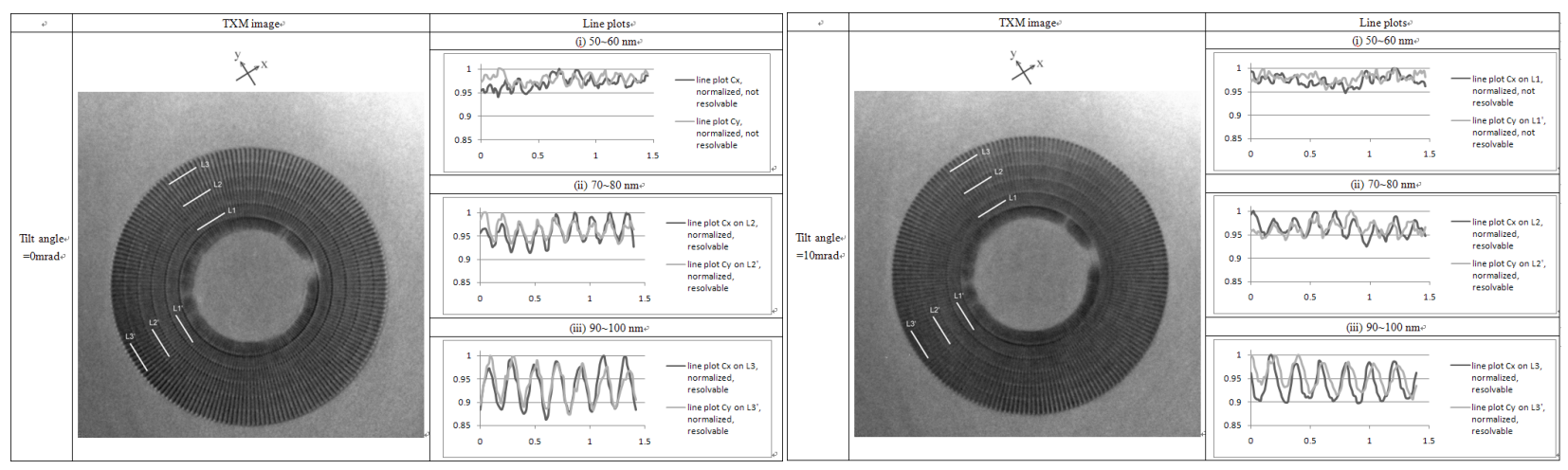

(a)

(b) 


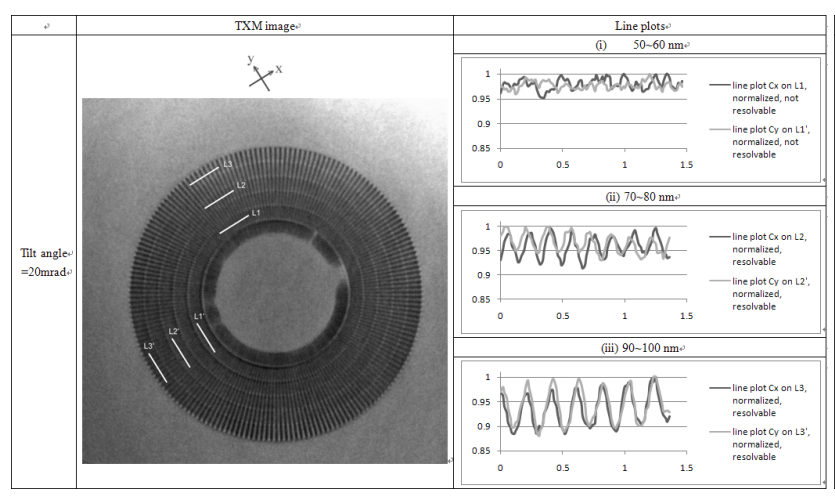

(c)

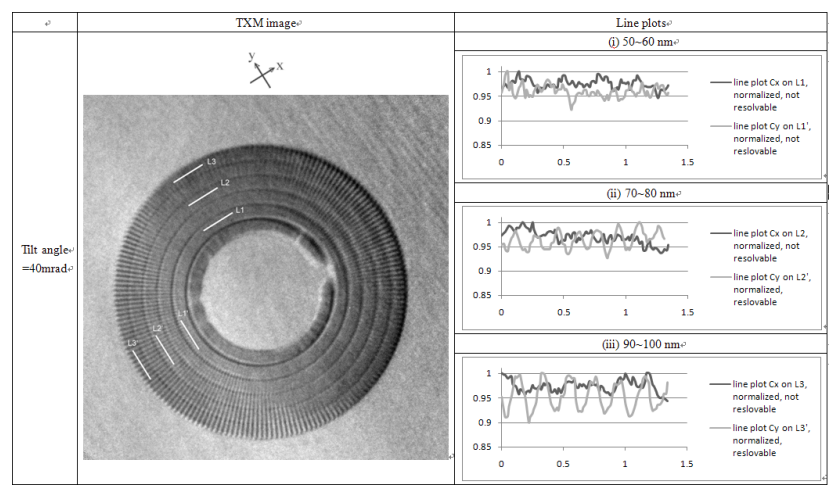

(e)

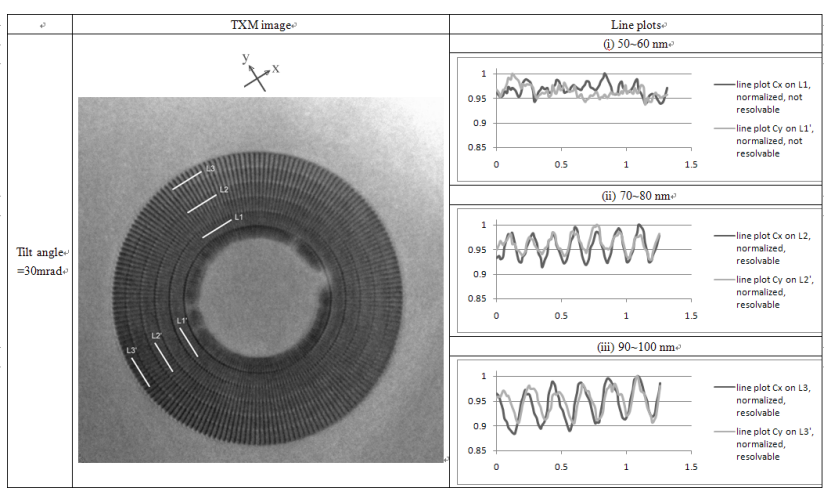

(d)

Table 4- 1 The experimental TXM image at tilt angle (a) $\theta=0$ mrad., (b) $\theta=10 \mathrm{mrad}$., (c) $\theta=20 \mathrm{mrad} .,(\mathrm{d}) \quad \theta=30 \mathrm{mrad}$., (e) $\theta=40$ mrad.

By the TXM images and the line plots in Table 4-1, we can see that, whether the zone plate is tilted or not, the patterns in the first and second zones are not resolvable, i.e., the resolution-limits of those TXM images are all larger than $60 \mathrm{~nm}$. Briefly, the TXM image does not change a lot when the zone plate is tilted from 0 to $30 \mathrm{mrad}$., as shown in Table 4-1 (a)-(d). However, when the zone plate is tilted by 40 mrad., the TXM image becomes worse; the patterns parallel to $\mathrm{x}$ axis are still resolvable, but the patterns parallel to $\mathrm{y}$ axis become blurred (the line plots on L2, L3 at tilt angle $=40$ mrad. are not periodic functions of distances, that is, the patterns which are parallel to the y axis on L2 and L3 can't be resolved when the zone plate is tilted by 40 mrad.), as show in Table 4-1 (e).

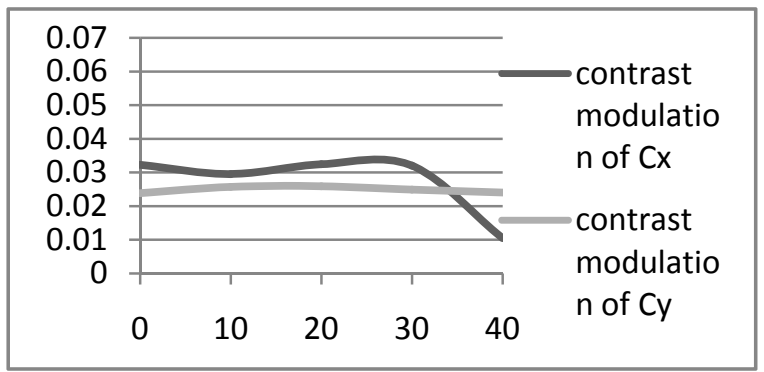

(a)

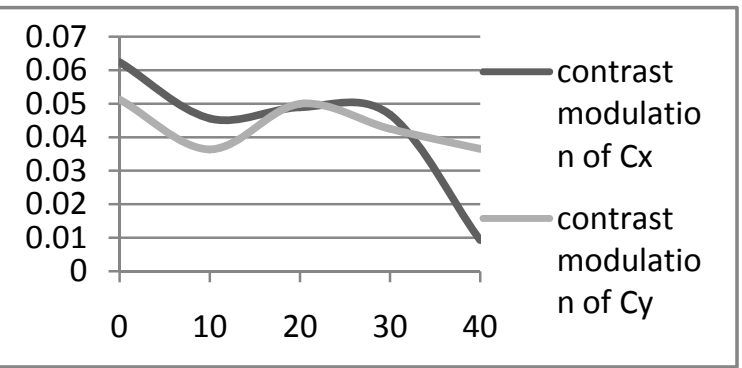

(b) 
Fig. 4-3 Contrast modulation v.s tilt angle (mrad.) (a) at spatial frequency between

$$
12.50 \mathrm{MHz} \sim 14.2857 \mathrm{MHz}\left(\frac{1}{70 \mathrm{~nm}} \sim \frac{1}{80 \mathrm{~nm}}\right)
$$

(b) at spatial frequency between $11.1111 \mathrm{MHz} \sim 10.0 \mathrm{MHz}\left(\frac{1}{90 \mathrm{~nm}} \sim \frac{1}{100 \mathrm{~nm}}\right)$.

In Fig. 4-3, the modulations of Cy do not change obviously as tilt angle increases (In Fig. 4-3 (a), the contrast modulation of $\mathrm{Cy}$ is between 0.02 and 0.03, and in Fig. 4-3 (b), it's between 0.035 and 0.052). The contrast modulations of $\mathrm{Cx}$ do not change a lot when the tilt angle is smaller than $30 \mathrm{mrad}$., but they decrease rapidly when the tilt angle is larger than $30 \mathrm{mrad}$. For this reason, we say that the maximum acceptable tilt angle is $30 \mathrm{mrad}$. Although the qualities of TXM images are affected by many reasons, and both the resolutions and contrasts of experimental results are worse than those of the simulation results, we can still see that the zone plate tilt affects the image quality.

\section{CONCLUSION}

The affections due to zone plate tilt on the TXM image can be observed in series of TXM images and their line plots in chapter 3 and 4 . According to section 2.2, the focal lengths $f_{x}{ }^{\prime}$ and $f_{y}{ }^{\prime}$ in horizontal and vertical directions of a tilted zone plate are different, and the effective transmission function of tilted zone plate will be quite different when the tilt angle is larger than $\tan ^{-1}\left(\frac{1}{\text { aspect ratio }}\right)$. As a result, astigmatism will occur and the ability of tilted zone plate to focus light in the horizontal direction will become worse, the image quality will be affected by zone plate tilt. By the simulation and experimental results, the expectance in section 2.2 is proved to be right. Zone plate tilt will make the TXM image becomes distorted and blurred, and both the simulation and experimental results indicate that the contrast of image becomes lower as the tilt angle increases.

\section{Acknowledgements}

This project was supported by the National Chiao Tung University and t National Synchrotron Radiation Research Center. The authors would like to thank Gung-Chian Yin for helpful discussions and suggestions.

\section{REFERENCES}

[1]. J. Kirz, "Phase zone plates for x rays and the extreme uv", Journal of the optical society of America. Volume 64, number 3, March 1974.

[2] W. Chao, B. D. Harteneck, J. A. Liddle, E. H. Anderson1 \& D. T. Attwood, "Soft X-ray microscopy at a spatial resolution better than 15nm", Nature, Vol 435|30 June 2005|doi:10.1038/nature03719

[3] Y Feng, M. Feser, A. Lyon, S. Rishton, X. Zeng, S. Chen, S. Sassolini, and W. Yun, "Nanofabrication of high aspect ratio 24 nm x-ray zone plates for x-ray imaging applications”, J. Vac. Sci. Technol. B, Vol. 25, No. 6, Nov/Dec 2007

[4.] G. Yin, M. Tang, Y. Song, F. Chen, F.W. Duewer, W. Yun, C. Ko, H.D. Shieh, and K. S. Liang, "Energy-tunable transmission $\mathrm{x}$-ray microscope for differential contrast 
imaging with near $60 \mathrm{~nm}$ resolution tomography", Appl. Phys. Lett. 88, 241115(2006)

[5]. G. Yin, M. Tang, Y. Song, F. Chen, F.W. Duewer, W. Yun, M. Feser, H.D. Shieh, and K. S. Liang, "30 nm resolution $\mathrm{x}$-ray imaging at $8 \mathrm{keV}$ using third order diffraction

of a zone plate lens objective in a transmission microscope", Appl. Phys. Lett. 89, 221122(2006)

[6] J. W. Goodman, [Introduction To Fourier Optics], the McGraw-Hill companies, New York, chapter 3. 